\title{
On Colorations Induced by Discrete Rotations
}

\author{
Bertrand Nouvel* and Éric Rémila \\ Laboratoire de l'Informatique du Parallélisme \\ UMR CNRS - ENS Lyon - INRIA 5668 \\ École Normale Supérieure de Lyon \\ 46, Allée d'Italie 69364 LYON CEDEX 07 - France \\ \{bertrand.nouvel, eric.remila\}@ens-lyon.fr
}

\begin{abstract}
We consider a non numerable family of colorations induced by discrete rotations. The symbolical dynamical system associated with the coloration is first explained. We introduce then a group that supports the dynamics of the system. The periodical cases are precised, they are induced by Pythagorean triples. Finally, a proof of the quasi-periodicity of the colorations, and a description of asymmetrical colorations conclude this paper.
\end{abstract}

\section{Introduction}

The search for discrete rotation algorithms that have similar properties as euclidian rotations (bijectivity, commutativity, etc.) was started by Andrès, Réveillès $([3]$, 8]), ten years ago. It remains today one of the most interesting -and hardest- problems of discrete geometry theory.

In this paper, we focused on the image of a single point's neighbors as transformed by discrete rotations. We have embedded this neighborhood information into each point as a color, therefore describing the transformation as a coloration of the grid $\mathbb{Z}^{2}$. This paper documents our investigations of local deformations, by the bias of a study of these colorizations.

We explain when and why the colorizations investigated are periodical,or asymmetric. We prove their quasi-periodicity, in the aim of laying the foundation for ongoing research based on their use.

\section{Definitions}

We denote by $\lfloor x\rfloor$ the integer part of $x$ : the integer such that $\lfloor x\rfloor \leq x<\lfloor x\rfloor+1$. The rounding function, or point-discretization function is defined as $[x]=\lfloor x+$ $0.5\rfloor$; it may be applied to vectors, components by components. We may notice that $[(-.5, .5)]=(\mathbf{0}, \mathbf{1})$. The composition of a function $f$ with the rounding function will be denoted by $[f]$. We define the application $\{$.$\} by \{x\}=x-[x]$. For a binary relation $r$ on set $E$, ie $\mathrm{r}$ is a subset of $E^{2}$, we denote $f(r)$ as the

\footnotetext{
* Financed via CIFRE by TF1 french television channel.
} 
set of all pairs $(f(x), f(y))$ with $(x, y)$ belonging to $r, r(x)$ is the set of all $y$ such that $(x, y)$ belongs to $r$. $R C(r)$, that stands for "relative coding", will refer to the binary relation 11 formed by all $(x, y-x)$ for $(x, y)$ belonging to $r$. In the real plane $\mathbb{R}^{2}, \mathbf{i}, \mathbf{j}$ will refer to the unit vectors $(\mathbf{1}, \mathbf{0})$ and $(\mathbf{0}, \mathbf{1})$. Assuming $\mathbf{v}$ is a vector, we denote the horizontal (resp. vertical) coordinate of $\mathbf{v}$ by $\mathbf{v}_{x}$ $\left(\mathbf{v}_{y}\right) . U$ denotes the application of $\mathbb{Z}^{2}$ that maps the point $\mathbf{p}$ to $\mathbf{p}+\mathbf{j}$. If $U(\mathbf{p})$ stands for "Up", one easily guesses what will be $D(\mathbf{p})$ for down, $L(\mathbf{p})$ for left and $R(\mathbf{p})$ for right. By $\mathcal{V}_{U}$ we mean the binary relation that links any point of the plane with its upward neighbor, $\left(\mathcal{V}_{U}=\left\{(x, U(x)) \mid x \in \mathbb{Z}^{2}\right\}\right)$. By $\mathcal{V}_{4}$ it's the 4-neighborhood (Von Neumann neighborhood) of a point in a the discrete plane $\mathbb{Z}^{2}$ that is denoted $\left(\mathcal{V}_{4}=\mathcal{V}_{L} \cup \mathcal{V}_{R} \cup \mathcal{V}_{D} \cup \mathcal{V}_{U}\right)$. When an application $f$ is bijective, $f^{-1}$ denotes the inverse application. If it is not one-to-one, $f^{\{-1\}}$ will denote the application that maps to $x$ the set of elements $y$ such that $f(y)=x$. If $\mathbf{x} \in \mathbb{R}^{2}$, the set $[[\mathbf{x}]]^{\{-1\}}$ will be called a discretization cell associated to $\mathbf{x}$; it is the unit square centered on $[x]$.

In this document, an arrow is an element of $\mathbb{A}=\{-1,0,1\}^{2}$. A coloration is an application that maps a point of $\mathbb{Z}^{2}$ into a finite set, which is called the colorset. (The elements of the colorset may be different from what we usually call colors, this is just a finite set). The colorset we are going to use across this document will be $\mathcal{P}(\mathbb{A})$, the set of subsets of $\mathbb{A}$. A coloration $C$ is deemed periodical, if and only if there exists two non colinear vectors $\mathbf{v}$ and $\mathbf{v}^{\prime}$ of $\mathbb{Z}^{2}$, such that for all $\mathbf{x} \in \mathbb{Z}^{2}, C(\mathbf{x})=C(\mathbf{x}+\mathbf{v})=C\left(\mathbf{x}+\mathbf{v}^{\prime}\right)$.

\section{Description of the Studied Colorations}

$r_{\alpha}$ is the euclidian rotation in the real euclidian plane. We consider the application that maps a point $\mathbf{x}$ of $\mathbb{Z}^{2}$ to the set of the arrows to its neighbors after a discrete rotation $\left[r_{\alpha}\right]$. Formally:

$$
G_{\alpha}(\mathbf{x}):=\bigcup_{\left(\mathbf{X}^{\prime} \in \mathcal{V}_{4}(\mathbf{x})\right)}\left\{\left[r_{\alpha}\right]\left(\mathbf{x}^{\prime}\right)-\left[r_{\alpha}\right](\mathbf{x})\right\}
$$

The information $G_{\alpha}$ can be also be affected to the discretization cell of $r_{\alpha}(x)$. For a discretization cell $c=[x]^{\{-1\}}$, we can also affect to $x$ the union of $G_{\alpha}(y)$ for all $y$ such that $r_{\alpha}(y) \in c$ : We denote by $G_{\alpha}^{\prime}$ the following coloration.

$$
\begin{gathered}
G_{\alpha}^{\prime}(\mathbf{x}):=\bigcup_{\left(\mathbf{y} \in\left[r_{\alpha}\right]^{\{-1\}}(\mathbf{x})\right)} G_{\alpha}(\mathbf{y}) \\
G_{\alpha}^{\prime}(\mathbf{x}):=\bigcup_{\left(\mathbf{y} \in\left[r_{\alpha}\right]^{\{-1\}}(\mathbf{x})\right)} \bigcup_{\left(\mathbf{y}^{\prime} \in \mathcal{V}_{4}(\mathbf{y})\right)}\left\{\left[r_{\alpha}\right]\left(\mathbf{y}^{\prime}\right)-\left[r_{\alpha}\right](\mathbf{y})\right\}
\end{gathered}
$$

$\left(G^{\prime}\right)$ 's construction is now detailed:

We consider the discrete lattice $\mathbb{Z}^{2}$. We rotate it, thus we get $r_{\alpha}\left(\mathbb{Z}^{2}\right)$, the rotated lattice is represented in dark on figure 1. a. On $\mathbb{Z}^{2}$, with dashed lines, we have also represented its dual, which corresponds to the Voronoi diagram of $\mathbb{Z}^{2}$, and divides the space into cells; These cells are the discretization cells. If a real point $\mathbf{v}$ is located in the cell associated with an integer coordinate point $\mathbf{p}$ the $[\mathbf{v}]=\mathbf{p}$. The exact behaviour of the relation on the border is induced from the behavior of the discretization operator [.].

\footnotetext{
${ }^{1}$ Note that we assume a "minus" operator has been defined between the elements.

${ }^{2}$ A lattice is here the couple $\left(\mathbb{Z}^{2}, \mathcal{V}_{4}\right)$.
} 


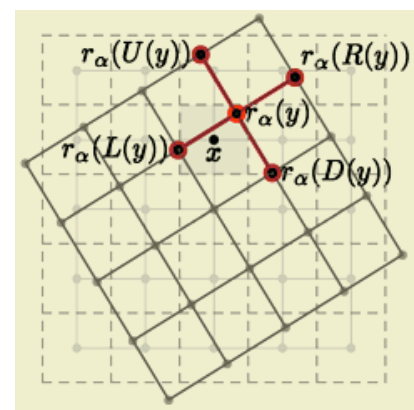

a)

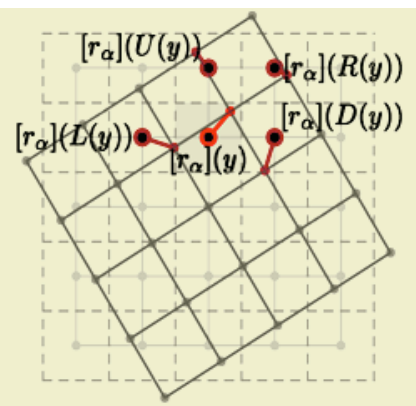

b)

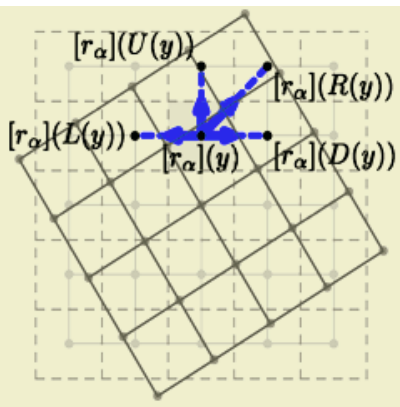

c)

Fig. 1. Construction of the coloration $G_{\alpha}(y)$ and $G_{\alpha}^{\prime}(x)$.

The neighborhood relation $\mathcal{V}_{4}$ on the rotated lattice is $r_{\alpha}\left(\mathcal{V}_{4}\right)$. (see figure. [1] a) We have overlined here the edges of the relation of $r_{\alpha}\left(\mathcal{V}_{4}\right)$ for one point of the rotated lattice. The relation is then discretized according to the underlying networl 3 . Each edge of the relation will be moved to the nearest integer point (see fig. 1 $1 \mathrm{~b}$ ), and thus we obtain $\left[r_{\alpha}\right]\left(\mathcal{V}_{4}\right)$. And we draw the edges of the corresponding relation $\left(\left[r_{\alpha}\right]\left(\mathcal{V}_{4}\right)(x)\right)$, see fig. 1 r), for the point $y$ that fall in a cell centered on $x$ after rotation.

We consider, $R C\left(\left[r_{\alpha}\left(\mathcal{V}_{4}\right)\right]\right)(x)$, the relative coding of the precedent relation. This is required in order to have a finite color set (which would be independant of the point considered in the relation). Moreover this provides the ability to compare, the colors of two points directly.

The cardinal of $\left[r_{\alpha}\right]^{\{-1\}}$ is at most 2. If the cardinal of $\left[r_{\alpha}\right]^{\{-1\}}(\mathbf{x})$ is zero then $\mathbf{x}$ is a hole in $G_{\alpha}^{\prime}$. If the cardinal of $\left[r_{\alpha}\right]^{\{-1\}}(\mathbf{x})$ is one then $\mathbf{x}$ is a normal point in $G_{\alpha}^{\prime}$. If the cardinal of $\left[r_{\alpha}\right]^{\{-1\}}(\mathbf{x})$ is two then $\mathbf{x}$ is a double point in $G_{\alpha}^{\prime}$. (see section 41).

When $\mathbf{x}$ is a double point in $G_{\alpha}^{\prime}$ then its associated code is the superposition (the union of the sets of arrows) of the code in $G$ that were affected to the points that transform to $\mathbf{x}$ via $\left[r_{\alpha}\right]$. An example of $G^{\prime}$ that denotes colors using arrows is presented on figure 2

\section{Elementary Properties}

During all this article, we are going to consider that $\alpha \in[0 \ldots \pi / 4]$. We are going now to present first some basic fundamental properties. Proofs have been omitted. They are available in [7].

- Two $\mathcal{V}_{4}$-neighbors can not be two holes.

- In $G_{\alpha}$ each color contains exactly of 3 or 4 different non null-arrows. If it is only 3 arrows then it means that there is one null-arrow 4 .

\footnotetext{
${ }^{3}$ The one that has not been rotated.

${ }^{4}$ And not that two arrows merge.
} 


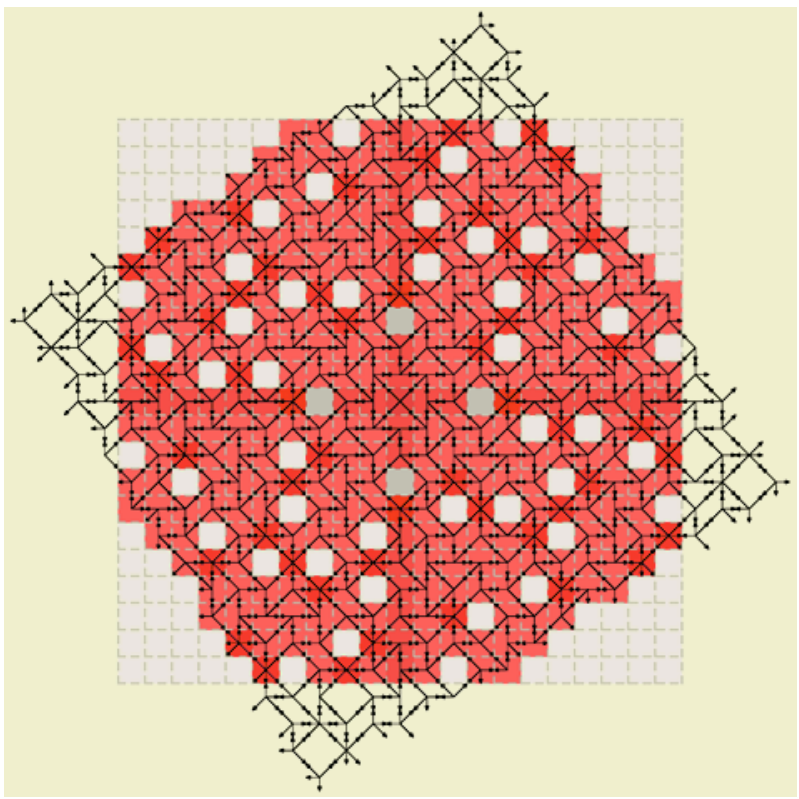

Fig. 2. Sample representation of the $G^{\prime}$ coloration for a random angle $(\alpha=$ $0.54977832 \mathrm{rad})$. The set of arrows inside a cell $c=[[\mathbf{p}]]^{-1}$ represents the value $G^{\prime}([p])$. The background color behind the arrows is function of the number of points that have an image in the discretization cell. Finally, the axis have also been a bit darkened.

- In $G_{\alpha}^{\prime}$ each color contains exactly of 0,4 , or 6 different non null-arrows. If it is 0 , the point considered is a hole, if it is 4 then it is a normal point, and finally, if there are 6 arrows, then it is a double point (issued from two 3 -arrows symbol in $G_{\alpha}$ ). It is important to note that two non null-arrows merge.

- The application $\alpha \mapsto G_{\alpha}$ is injective from the $[0 \ldots \pi / 4]$ to $\mathcal{P}(\mathbb{A})^{\mathbb{Z}^{2}}$.

- The colorations admit a central symmetry for all angles except a numerable set called special angles, which will be presented later in this text.

- In $G$, there exists a application that maps a color, to the arrow that denotes only the position of a specified neighbor $(U(\mathbf{x}), R(\mathbf{x}), L(\mathbf{x})$ or $D(\mathbf{x}))$.

\section{$5 \quad$ Algebraic Properties}

A window is the products of intervals on $\mathbb{Z}$. We will denote by $\left[\mathbf{p}_{x}, \mathbf{p}_{x}+\right.$ $\mathbf{s}_{x}\left[\mathbb{Z} \times\left[\mathbf{p}_{y}, \mathbf{p}_{y}+\mathbf{s}_{y}\left[\mathbb{Z}\right.\right.\right.$ the window located at $\mathbf{p}$ and of size $\mathbf{s}$; it contains $\mathbf{s}_{x} \cdot \mathbf{s}_{y}$ points of $\mathbb{Z}^{2}$.

We define a pattern as a function of a window $\left[0, \mathbf{s}_{x}\left[\mathbb{Z} \times\left[0, \mathbf{s}_{y}[\mathbb{Z}\right.\right.\right.$ to the colorset $Q$. Let $C$ be a coloration and $\pi$ a pattern of size $\left(\mathbf{s}_{x}, \mathbf{s}_{y}\right)$. If there exists a point

\footnotetext{
${ }^{5}$ We denote intervals on $\mathbb{Z}$ by $[a, b[\mathbb{Z}$.
} 


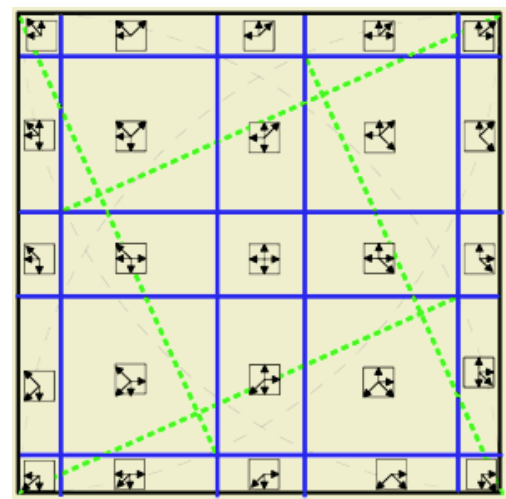

$0 \leq \alpha<\pi / 6$

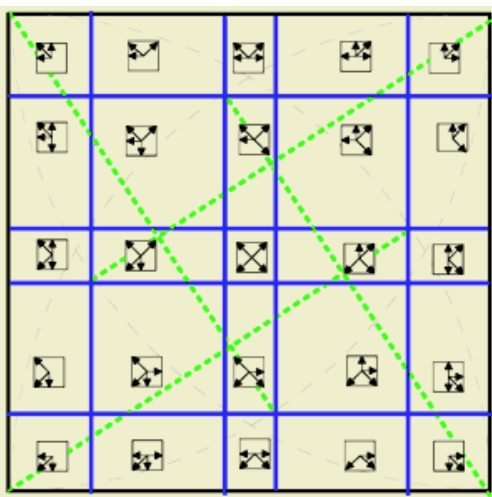

$\pi / 6<\alpha \leq \pi / 4$

Fig. 3. In the figure above, we see an example of a map that binds a frame in the torus to it's associated symbol. If the image of a point $p$ by $\left\{r_{\alpha}\right\}$ is in a the frame $I_{s}$, then $G_{\alpha}=s$. Of course, the arrows in the symbol indicate the location of the cells where the rotated neighbors of that point are.

$\mathbf{p} \in \mathbb{Z}^{2}$ such that for all $0 \leq \mathbf{t}_{x}<\mathbf{s}_{x}$ and for all $0 \leq \mathbf{t}_{y}<\mathbf{s}_{y}$, we have $C(\mathbf{p}+\mathbf{t})=$ $\pi(\mathbf{t})$, then we say that the pattern $\pi$ appears (at $\mathbf{p}$ ) in the coloration $C$.

We define the torus $\mathbb{T}=(\mathbb{R} / \mathbb{Z})^{2}$, we will often use $\{$.$\} as a projection on$ this torus. And generally when we represent it we will represent it from $-\frac{1}{2}$ to $\frac{1}{2}$ such that the $\{0\}$ is placed at the center of the square representing the torus. Therefore we can identify this representation of the torus and a discretization cell. We define a frame as products of projections of real intervals on the torus $\mathbb{T}$.

The following theorem is fundamental, due to the fact that it will be at the basis of all the analysis of the colorations.

Theorem 1 (Fundamental Theorem). There exists a partition $I_{1}, \ldots, I_{n}$ of the torus into a subset of generally 25 frames of the torus $\mathbb{T}$ such that for all $G_{\alpha}(\boldsymbol{x})$ there exists a frame $f_{i}$ such that for all $\boldsymbol{x}^{\prime}, G_{\alpha}\left(\boldsymbol{x}^{\prime}\right)=G_{\alpha}(\boldsymbol{x})$ if and only if $\left\{r_{\alpha}\left(\boldsymbol{x}^{\prime}\right)\right\} \in f_{i}$.

\section{Proof. (elements of proof)}

Without lost of generality, we will focus only on the position of the right neighbor $\mathbf{p}+\mathbf{i}$ of point $\mathbf{p}$, and only on the question: "Is this neighbor mapped by $\left[r_{\alpha}\right]$ on the same vertical coordinate as $\mathbf{p}$ or not?"

We have $\left[r_{\alpha}\right](\mathbf{p}) \cdot \mathbf{i}=\left[r_{\alpha}\right](\mathbf{p}+\mathbf{i}) \cdot \mathbf{i}$, if and only if $\left\{r_{\alpha}\right\}(\mathbf{p}) \cdot \mathbf{i}+\frac{1}{2}<1-\cos (\alpha)$. Therefore that splits the torus in two frames according to a vertical line located at $x=\frac{1}{2}-\cos (\alpha)$.

We will obtain the same kind of results for the other quotient colorations. 6 . Finally we obtain of 4 vertical lines and 4 horizontal lines that splits of the torus (generally in 25 parts when no lines are merged).

\footnotetext{
${ }^{6}$ Considering the positions of others neighbors $(U(\mathbf{x}), L(\mathbf{x}), D(\mathbf{x}), R(\mathbf{x}))$, relatively to me in one of the two directions $\mathbf{i}$ or $\mathbf{j}$.
} 
The partitions we present (fig. 3) are easily built by attaching a rotated unit vector to each corner of the square, and by splitting the unit square vertically and horizontally on the other end of that vector. The construction lines are dashed.

Corollary 1 (Fundamental theorem for $G^{\prime}$ ). There exists a partition $I_{1}, \ldots$, $I_{n}$ and $I_{i_{1}}^{\prime}, I_{i_{2}}^{\prime}, I_{i_{3}}^{\prime}, I_{i_{4}}^{\prime}$ of the torus $\mathbb{T}$ into frames such that $G_{\alpha}(x)=n$ if and only if $\forall y \in r_{\alpha}^{\{-1\}}(x),\left(\left\{r_{\alpha}\right\}(y) \in I_{n}\right.$ or $\left.\left\{r_{\alpha}\right\}(y) \in I_{n}^{\prime}\right)$.

The proof relies on similar ideas.

Theorem 2 (Fundamental theorem for patterns in $G_{\alpha}$ (resp. $\left.G_{\alpha}^{\prime}\right)$ ). To any pattern $\pi$ that appears in the coloration $G_{\alpha}$ (resp. $\left.G_{\alpha}^{\prime}\right)$, it is possible to associate a frame $I_{\pi}$, such that the pattern $\pi$ appears in $G_{\alpha}$ at a position $\boldsymbol{p}$ if and only if $\{p\} \in I_{\pi}$.

Moreover, for all size vector $s$, the set of patterns of size $s$ partition the torus.

Proof. We consider a rectangular pattern $\pi_{1}$ appearing at $\mathbf{p}_{0}$ in a coloration $G_{\alpha}$ and of size s. The pattern appears: for all $\mathbf{t}$, with $0 \leq \mathbf{t}_{x}<\mathbf{s}_{x}$ and $0 \leq$ $\mathbf{t}_{y}<\mathbf{s}_{y}$, we get $G_{\alpha}(\mathbf{p}+\mathbf{t})=\pi(\mathbf{t})$. Using the fundamental theorem,for all $\mathbf{t}$, with $0 \leq \mathbf{t}_{x}<\mathbf{s}_{x}$ and $0 \leq \mathbf{t}_{y}<\mathbf{s}_{y},\left\{r_{\alpha}(\mathbf{p}+\mathbf{t})\right\} \in I_{f(\pi(\mathbf{t}))}$. In addition, $\{$.$\} is a morphism for addition, therefore for all \mathbf{t}$, with $\mathbf{t}_{x}<\mathbf{s}_{x}$ and $\mathbf{t}_{y}<\mathbf{s}_{y}$, $\left(\left\{r_{\alpha}(\mathbf{p})\right\}+\left\{r_{\alpha}(\mathbf{t})\right\}\right) \in\left\{I_{f(\pi(\mathbf{t}))}\right\}$, and thus $\left\{r_{\alpha}(\mathbf{p})\right\} \in\left\{I_{f(\pi(\mathbf{t}))}-r_{\alpha}(\mathbf{t})\right\}$. Finally we pose $I_{\pi_{1}}=\cap_{0<\mathbf{t}_{x}<\mathbf{s}_{x}} \cap_{0<\mathbf{t}_{y}<\mathbf{s}_{y}}\left\{I_{f(\pi(\mathbf{t}))}-r_{\alpha}(\mathbf{t})\right\}$. The last statement rewrites into $\pi_{1}$ appears at $\mathbf{p}$, if and only if $\left\{r_{\alpha}(\mathbf{p})\right\} \in I_{\pi_{1}} . I_{\pi_{1}}$ is a frame, and we know that it is not empty 7 , therefore we know that it has a non null area.

Moreover, it is obvious that for differents two patterns $\pi_{1}, \pi_{2}$ of the same size $\mathbf{s}, f_{\pi_{1}} \cup f_{\pi_{2}}=\emptyset$. Finally, if there can not be a point of the torus without a pattern of size $\mathbf{s}$ associated, therefore the set of patterns of size $\mathbf{s}$ partition the torus.

Corollary 2 (Fundamental theorem for $G^{\prime}$ (extension)). There exists frames that signal if there is hole on a neighboring cell.

\section{Study of Periodicity and Quasiperiodicity}

A coloration $C$ is quasi-periodical if and only if for all patterns $\pi$ that appears in $C$, there exists a size of window $\mathbf{s}$, such that $\pi_{1}$ appears in all windows of size s.

We introduce the two vectors $\mathbf{i}_{\alpha}=r_{\alpha}(\mathbf{i})$ and $\mathbf{j}_{\alpha}=r_{\alpha}(\mathbf{j})$. We consider $\mathcal{G}$ the abelian group generated by: $\mathbb{Z} \mathbf{i}+\mathbb{Z} \mathbf{j}+\mathbb{Z} \mathbf{i}_{\alpha}+\mathbb{Z} \mathbf{j}_{\alpha}$. We note that $\mathcal{G}$ is invariant by integer translation and rotation of angle $\pi / 2$.

$\mathcal{G}^{\prime}$ is the subgroup of $\{G\}$ of $\mathbb{T}$. The subgroup is generated by $\left\{\mathbf{i}_{\alpha}\right\}$ and $\left\{\mathbf{j}_{\alpha}\right\}$.

$\overline{7 \text { It appears at }} \mathbf{p}_{0},\left\{r_{\alpha}\left(\mathbf{p}_{0}\right)\right\} \in I_{\pi_{1}}$. 
The application $\{$.$\} is a one-to-one map from \mathcal{G}^{\prime}$ to $\mathcal{G} \cap F$, where $F$ is a unit window $\left[\beta, \beta+1\left[\times\left[\gamma, \gamma+1\left[\right.\right.\right.\right.$ in $\mathbb{R}^{2}$. Let $\mathbf{p} \in \mathbb{R}^{2}, \mathbf{p} \in \mathcal{G}$ if and only if $\{\mathbf{p}\} \in \mathcal{G}^{\prime}$. $\mathcal{G}^{\prime}$ can equivalently be introduced as the set of points of $\left\{r_{\alpha}(\mathbf{p})\right\}$ for all $\mathbf{p} \in \mathbb{Z}^{2}$.

We are going to investigate the structure of $\mathcal{G}$, we define the set $S_{\mathcal{G}}$ so $\{\|v\|, v \in \mathcal{G} \backslash\{(0,0)\}\}$. We can consider the two following cases: either $\inf \left(S_{\mathcal{G}}\right)=$ $l_{0}$, and $l_{0} \neq 0$, either $\inf \left(S_{\mathcal{G}}\right)=0$.

\subsection{The Case $\inf \left(S_{\mathcal{G}}\right)=l_{0}$, and $l_{0} \neq 0$, the Discrete Case}

We are in the case where the lower bound of $S_{\mathcal{G}}$ is not null.

Proposition 1. Let $l_{0}$ be this lower bound. There exists a vector e of $G$ which has $l_{0}$ as norm.

Proof. We consider the compact crown 8 of depth $\left[l_{0}, l_{0}+\epsilon\right]$. There exists a sequence of vectors of $G$ such that the norms of the vectors converges to $l_{0}$, and thus a vector $v_{0}$. There exists an extracted subsequence that converges. The sequence of the difference between two consecutive terms of this sequence converge to 0 , if the bound will not have been reached, from the previous sequence we would extract an arbitrary small element. Thus we would have a contradiction.

Proposition 2. We define $\boldsymbol{e}^{\prime}=r_{\pi / 2}(\boldsymbol{e})$; we have $G=\mathbb{Z} \boldsymbol{e}+\mathbb{Z} \boldsymbol{e}^{\prime}$. In addition, $\mathcal{G}^{\prime}$ is finite.

Proof. Let's assume that there is an element $k$ of $\mathcal{G}$ outside $\mathcal{G}=\mathbb{Z} \mathbf{e}+\mathbb{Z} \mathbf{e}^{\prime}$. We are in a two dimensional space therefore, that would generate a smallest vector in $G$, which would contradict the minimality of e. $\mathcal{G}^{\prime}$ is finite since $G \cap \mathbb{T}$ is finite and $\{$.$\} is a one-to-one map to \mathcal{G}^{\prime}$.

Theorem 3. If $\mathcal{G}^{\prime}$ is finite then the colorations $G_{\alpha}$ and $G_{\alpha}^{\prime}$ are periodical.

Proof. Since $G^{\prime}$ is finite, there exists a constant $K$ such that $K\{\mathbf{e}\}=0$ in $\mathcal{G}^{\prime}$.

Let's stands that $\mathbf{e}=a_{\mathbf{e}} \mathbf{i}+b \mathbf{e} \mathbf{j}+c_{\mathbf{e}} \mathbf{i}_{\alpha}+d_{\mathbf{e}} \mathbf{j}_{\alpha}$. Which gives to us: $\{\mathbf{e}\}=$ $c_{\mathbf{e}}\left\{\mathbf{i}_{\alpha}\right\}+d_{\mathbf{e}}\left\{\mathbf{j}_{\alpha}\right\}$. Moreover $c_{e}^{2}+d_{e}^{2} \neq 0$ if and only if $\mathcal{G}$ is not included in $\mathbb{Z}^{2}$ (which does not occur if we suppose $\alpha \neq 0$ module $\pi / 2$ ).

Let $x$ be a point of $\mathbb{Z}^{2} \cdot\left\{r_{\alpha}\left(\mathbf{x}+K c_{\mathbf{e}} \mathbf{i}+K d \mathbf{e j}\right)\right\}=\left\{r_{\alpha}(\mathbf{x})+K c_{\mathbf{e}} \mathbf{i}_{\alpha}+K d_{\mathbf{e}} \mathbf{j}_{\alpha}\right\}=$ $\left\{r_{\alpha}(\mathbf{x})\right\}+K c_{\mathbf{e}}\left\{\mathbf{i}_{\alpha}\right\}+K d_{\mathbf{e}}\left\{\mathbf{j}_{\alpha}\right\}=\left\{r_{\alpha}(\mathbf{x})\right\}+K\{\mathbf{e}\}=\left\{r_{\alpha}(\mathbf{x})\right\}$. This proves that coloration $G$ is periodical of period $K c \mathbf{e} \mathbf{i}+K d \mathbf{e j}$. Using $\mathbf{e}^{\prime}$ in a similar process, we find that the coloration is periodical of period: $-K d \mathbf{e} \mathbf{i}+K c \mathbf{e j}$. Therefore the coloration is periodical (using the fundamental theorem 1).

$\mathcal{G}^{\prime}$ is assumed to be discrete, the coloration is periodical. There exists constants $K_{1}$ and $K_{2}$ such that $K_{1}\{\mathbf{e}\}+K_{2}\left\{\mathbf{e}^{\prime}\right\}=\{\mathbf{i}\}$. One may project the vector $\mathbf{e}$ and $\mathbf{e}^{\prime}$ on one dimension of the space. Hence, one may view this equation as

${ }^{8}$ the set: $\overline{\mathcal{B}_{l_{0}+\epsilon} \backslash \mathcal{B}_{l_{0}}}$, where $\mathcal{B}_{x}$ is the opened ball of radius $x$ 
one where the two unknowns are $\cos (\alpha)$ and $\sin (\alpha)$ (all others parameters are integers). This implies that $\cos (\alpha)$ and $\sin (\alpha)$ are both rationnal. And thus that $\alpha$ is a Pythagorean angle 9 . There are particular cases of Pythagorean, for which discrete rotation is bijective, for more details have a look at [6]. Conversely, when $\alpha$ is Pythagorean, $\cos (\alpha)$ and $\sin (\alpha)$ are rationnal, $\mathcal{G}$ is discrete, all points are separated and $\mathcal{G}^{\prime}$ is finite.

\subsection{The Case Where $\inf \left(S_{\mathcal{G}}\right)=0$, the Dense Case}

We are in the case were the lower bound of $S_{\mathcal{G}}$ is null. For all $\epsilon>0$, there exists a vector $\mathbf{v}_{\epsilon}$ of $\mathcal{G}$ such that $\left\|\mathbf{v}_{\epsilon}\right\| \leq \epsilon$. Thus, there exists in $G$ a grid formed by squares of side less than $\epsilon$. In addition $\mathcal{G}$ is a dense subset of $\mathbb{R}^{2}$.

Theorem 4. For all angle $\alpha \in[0 \ldots \pi / 4]_{\mathbb{R}}$, if $\inf \left(S_{\mathcal{G}}\right)=0$ then $G_{\alpha}$ is quasiperiodical.

Proof. We consider $\mathcal{G}_{\epsilon}=[-1 \ldots 1]_{\mathbb{R}}^{2} \cap\left(\mathbf{e}_{\epsilon} \mathbb{Z}+\mathbf{e}_{\epsilon}^{\prime} \mathbb{Z}\right)$ the set generated by linear composition of $\mathbf{e}_{\epsilon}$ and $\mathbf{e}_{\epsilon}^{\prime}$, (orthogonal vectors in $\mathcal{G}$ with a norm less or equal to $\epsilon$ ), and for which the components on $\mathbf{i}, \mathbf{j}$ are less than 1 . It has the property that for all elements $\mathbf{z}$ of $\mathcal{G}^{\prime}$ and $\mathbf{x}$ of $\mathcal{G}$, there exists an element $\mathbf{y}$ of $\mathcal{G}_{\epsilon}$ such $\{\mathbf{x}+\mathbf{y}\}$ is at a distance at most $\epsilon$ of $\mathbf{z}$. All elements $\mathbf{y}$ of $\mathcal{G}_{\epsilon}$ may be written so $\mathbf{y}=a_{\mathbf{y}} \mathbf{i}+b_{\mathbf{y}} \mathbf{j}+c_{\mathbf{y}} \mathbf{i}_{\alpha}+d_{\mathbf{y}} \mathbf{j}_{\alpha}$.

$\mathcal{G}_{\epsilon}$ is finite, so there exists constants $C_{\epsilon}$ and $D_{\epsilon}$, such that for all $y \in \mathcal{G}_{\epsilon}$, we have $\left|c_{\mathbf{y}}\right|<C_{\epsilon}$ and $\left|d_{\mathbf{y}}\right|<D_{\epsilon}$. Consider now a window $F_{\epsilon, x}$ of size $2 C_{\epsilon} \times 2 D_{\epsilon}$ centered on $x$. For all element $y$ of $G_{\epsilon}$, there exists an integer element $\mathrm{x}^{\prime}$ of this window such that $\left\{r_{\alpha}\right\}\left(\mathbf{x}^{\prime}\right)-\left\{r_{\alpha}\right\}(\mathbf{x})=\{\mathbf{y}\}$. One may take $\mathbf{x}^{\prime}=\mathbf{x}+c_{\mathbf{y}} \mathbf{i}+d_{\mathbf{y}} \mathbf{j}$. In other words: $\left\{r_{\alpha}\right\}\left(F_{\epsilon, \mathbf{x}}\right)$ contains $\left\{r_{\alpha}\right\}(\mathbf{x})+\left\{\mathcal{G}_{\epsilon}\right\}$.

Let $\mathbf{x}$ be a point of $\mathbb{Z}^{2}$, and $\pi$ a pattern of the coloration, this pattern $\pi$ appears at $\mathbf{p}$ if and only if $\left\{r_{\alpha}\right\}(\mathbf{p})$ is in fixed frame $Z_{M}$ of $\mathbb{T}$ (theorem 2). We note that this $Z_{M}$ contains a frame of size $\mu \times \mu$ (without loss of generality, we may suppose it square).

Thus if we consider $\epsilon=\mu / 2$, then we ensure that an element $\left\{\mathbf{y}+r_{\alpha}(\mathbf{x})\right\}$ belongs to $Z_{M}$ (because there will be one in any square of dimension $\mu \times \mu$ ), which will provide us an element $\mathbf{x}^{\prime}$ of $F_{\epsilon, \mathbf{x}}$ is such that $\left\{r_{\alpha}\right\}\left(\mathbf{x}^{\prime}\right)$ is in $Z_{M}$. This proves quasi-periodicity of $G_{\alpha}$.

More precisions on the quasiperiodicity can be added considering the continued fraction development of $\cos (\alpha)$ and $\sin (\alpha)$, then using the three distance theorem (see [9], a good survey is also [1]).

\section{Asymmetrical Cases}

Let $\alpha$ and $\alpha^{\prime}$ be two angles, we define a distance on colorations considering the distance of the first point that differs in the two colorations:

\footnotetext{
${ }^{9}$ A Pythagorean angle is an angle $\alpha$ which may be written $\alpha \equiv \arctan \left(\frac{a}{b}\right)$, where $(a, b, c)$ is Pythagorean triples, ie $a, b, c \in \mathbb{N}$ and $a^{2}+b^{2}=c^{2}$. Pythagorean angles are angles for which both cosinus and sinus are rational.
} 


$$
d\left(G_{\alpha}, G_{\alpha^{\prime}}\right)=2^{-\inf \left\{d \in \mathbb{R},\|\mathbf{p}\|=d \text { and } \mathbf{p} \in \mathbb{Z}^{2} \mid G_{\alpha}(\mathbf{p}) \neq G_{\alpha^{\prime}}(\mathbf{p})\right\}}
$$

We introduce the following function: st $:[0, \pi / 4]_{\mathbb{R}} \rightarrow \mathbb{R}^{+}$which to $\alpha$ associates $\lim _{\epsilon \rightarrow 0} \sup _{\left.\alpha^{\prime} \in\right] \alpha-\epsilon, \alpha+\epsilon[} d\left(G_{\alpha}, G_{\alpha^{\prime}}\right)$. An angle $\alpha$ is stable if st $(\alpha)=0$. An angle $\alpha$ is unstable if $s t(\alpha)=k, k \in \mathbb{R}^{+} \backslash\{0\}$. Also we say that the configuration is unstable. A configuration is special, if and only if, there exists a point $\mathbf{p}$ of $\mathbb{Z}^{2}$ that is mapped after transformation on the border of a discretization cell.

Proposition 3. For an angle $\alpha$, the following propositions are equivalent:

- $G_{\alpha}$ is special

- $G_{\alpha}$ is unstable

$-G_{\alpha}$ is asymmetric

Proof. The only interesting case is: unstable $\Rightarrow$ special, all others implications are immediate. If it is unstable, then it means that there is point $\mathbf{p}$ of $r_{\alpha}\left(\mathbb{Z}^{2}\right)$ such that any slight variation in $\alpha$ would make change the cell's it belongs to. Therefore $r_{\alpha}(\mathbf{p})$ is on an edge of discretization cell. By definition, it means that $G_{\alpha}$ is a special configuration.

As corollary we get that the pythagorean configurations are stable.

\section{Conclusion}

The quasi-periodicity of the colorations studied provide us with information on the constraints of an algorithm that could generate the colorations.

It may be important to say that there is a simple algorithm which takes for input a point $\mathbf{p}$ of the plane and the coloration induced by a discrete rotation of angle $\alpha$, and returns the image of $\mathbf{p}$ by discrete rotation.

As long as no finite memory automaton may generate a strictly quasiperiodical sequence, and in order to provide the required input to the algorithm, it will be necessary to study in depth the simple cases formed by the periodical and special colorations.

To answer the previous question, it is also possible to explore these colorations from a combinatoric viewpoint basing our researches on studies of bi-sturmian sequences(see [10], 5], [4]). Discrete rotations may also be considered as an alternative interpretation of patterns issued from a particular class of 4-to-2 quasi-periodic tilings.

Finally, one may define similar colorations in order to study any quasiisometry - particularly, quasi-affine transformations. In ongoing research, we will compare these colorations with the ones generated by other kinds of rotations, such as E. Andrès's rotations (see 2]) or variants of rotation by circles. 


\section{References}

1. Pascal Alessandri and Valerie Berthé. Three distance theorems and combinatorics on words. L'enseignement mathématique, 2(44):103-132, 1998.

2. Eric Andres. The quasi-shear rotation. In 6th Int. Workshop on Discrete Geometry for Computer Imagery, Lyon (France), volume 1176 of Lecture Notes for Computer Science, Nov 1996. pp.307-314.

3. Eric Andres. Habilitation à diriger des recherches: Modélisation analytique discrète d'objets géometriques, 2000.

4. Pierre Arnoux, Valérie Berthé, Hiromi Ei, and Shunji Ito. Tilings, quasicrystals, discrete planes, generalized substitutions, and multidimensional continued fractions. Discrete Mathematics and Theoretical Computer Science Proceedings, 2001.

5. Valérie Berthé and Laurent Vuillon. Suites doubles de faible complexité. Journal de Théorie des Nombres de Bordeaux, 12:179-208, 2000.

6. Marie-André Jacob and Eric Andrès. On discrete rotations. In Discrete Geometry for Computer Imagery, 1995.

7. Bertrand Nouvel. Action des rotations sur le voisinage dans le plan discret. Master's thesis, ENS-Lyon, 2002.

8. J.P. Réveillès. Géométrie disrète, Calcul en nombre entiers, et Algorithmique. PhD thesis, ULP, 1991.

9. V.T. Sós. On the distribution $\bmod 1$ of the sequence $n \alpha$. Ann. Univ. Scient. Budapest., Eötvös Sect. Math. 1, 1958.

10. Laurent Vuillon and Valérie Berthé. Tilings and rotations on the torus: a twodimensional generalization of sturmian sequences. Discrete Mathematics, 2000. 